\title{
Schematic Models for Active Nonlinear Microrheology
}

\author{
M V Gnann, ${ }^{a}$ I Gazuz,${ }^{a \ddagger}$ A M Puertas, ${ }^{* b}$, M Fuchs, ${ }^{a}$ and Th Voigtmann ${ }^{a, c, d}$
}

\author{
Received Xth XXXXXXXXXX 20XX, Accepted Xth XXXXXXXXX 20XX \\ First published on the web Xth XXXXXXXXXX $200 X$ \\ DOI: $10.1039 / b 000000 x$
}

\begin{abstract}
We analyze the nonlinear active microrheology of dense colloidal suspensions using a schematic model of mode-coupling theory. The model describes the strongly nonlinear behavior of the microscopic friction coefficient as a function of applied external force in terms of a delocalization transition. To probe this regime, we have performed Brownian dynamics simulations of a system of quasi-hard spheres. We also analyze experimental data on hard-sphere-like colloidal suspensions [Habdas et al., Europhys. Lett., $2004,67,477]$. The behavior at very large forces is addressed specifically.
\end{abstract}

\section{Introduction}

Microrheology is a developing technique to acquire local information on the viscous and elastic properties of complex fluids and soft matter. ${ }^{[1.4}$ In active microrheology one uses a mesoscopic colloidal probe particle manipulated by an external driving mechanism such as laser tweezers or magnetic forces, and measures the response of that particle to the driving. The two major complications in making sense of the obtained data are, firstly, that one usually deals with suspending host liquids that have mesoscopic structure on length scales comparable to the size of the probe, calling into question simplifying assumptions treating the host liquid as a continuum. $\frac{5}{5}$ Second, even moderate external driving is sufficient to enter the nonlinear-response regime of soft host liquids. On the other hand, if one is able to understand the implications of this situation, the technique in principle gives access to detailed information about structure-dynamics relationships of complex soft matter. This makes active microrheology an ideal tool to probe, e.g., cellular environments. $\frac{1667}{}$

Here, we focus on the application of constant-forcing active microrheology to colloidal glass formers, different from variable forcings such as parabolic traps moving at constant velocity,, 8 or where the tracer moves at a constant speed. .910 Approaching the glass transition, the dynamics of the host liquid becomes increasingly slow, heterogeneous, and stretched over many orders of magnitude in time. Microrheology might, if properly understood, provide valuable insights into the microscopic origins of this slow structural relaxation dynam-

\footnotetext{
${ }^{a}$ Fachbereich Physik, Universität Konstanz, 78457 Konstanz, Germany.

${ }^{b}$ Departamento de Física Aplicada, Universidad de Almería, 04.120 Almería, Spain.E-mail: apuertas@ual.es

${ }^{c}$ Institut für Materialphysik im Weltraum, Deutsches Zentrum für Luft- und Raumfahrt (DLR), 51170 Köln, Germany.

${ }^{d}$ Zukunftskolleg, Universität Konstanz, 78457 Konstanz, Germany.

$\ddagger$ Present address: Leibnitz Institute of Polymer Research Dresden, Hohe Straße 6, 01069 Dresden, Germany
}

ics. $11-13$ The slow relaxation processes render highly nonlinear the relation between the measured steady-state velocity of the probe and the externally applied force. A pronounced superlinear rise in the velocity is observed for forces far exceeding the scale set by thermal energy and the particle size. ${ }^{[2]}$ This force threshold can be interpreted as the strength of nearestneighbor cages that are broken by strong forcing before they can relax due to structural relaxation.

We have recently presented a microscopic theory of nonlinear force-driven active microrheology for dense colloidal suspensions: ${ }^{14}$ based on an integration-through transients (ITT) framework and mode-coupling approximations, equations for the microscopic friction coefficient $\zeta\left(F^{\mathrm{ex}}\right)$ were obtained, defined by the steady-state relationship

$$
\zeta\langle\vec{v}\rangle_{t \rightarrow \infty}=\vec{F}^{\mathrm{ex}}
$$

where angular brackets denote the steady-state ensemble average reached at long times. These equations have a fully microscopic foundation as they are based on the Smoluchowski equation for the colloidal system (neglecting hydrodynamic interactions). They only require the equilibrium static structure factor as input. This, however, makes them also rather complicated to solve. In order to understand generic features of the equations, we have devised schematic models, i.e., adhoc simplifications of the original equations that are much easier to solve. The simplification essentially amounts to dropping spatial information (wave-vector dependences), at the cost of introducing a limited number of fitting parameters that replace the static-structure-factor input. One such schematic model was already presented earlier, 14 but this model did not capture some features of the high-force limit of the dynamics observed in experiment and simulation. In the following, we present an extended model that levies this limitation by taking into account separately the symmetry-breaking direction along the external force, and directions perpendicular to this. We are thus able to discuss the qualitatively different be- 
havior of probe fluctuations along those directions, and their effects on the friction in the steady-state probe velocity. The model is checked with computer simulations of a Brownian system, which we perform, and experiments taken from Habdas et al. ${ }^{12}$ The model correctly describes the qualitative features of the friction coefficient as a function of density and external forcing, and reproduces them quantitatively with a sensible choice of the parameters for both the simulations and experiments. The tracer position correlation function, a key quantity in the theory, behaves similarly in simulation and theory.

\section{Computer Simulation}

We performed molecular-dynamics simulations for quasihard-sphere particles governed by a Langevin equation, in the following referred to as Brownian dynamics (BD) simulations as hydrodynamic interactions are neglected. The system is polydisperse to avoid crystallization (flat distribution of radii, with half-width $10 \%$ of the average radius), and the interaction potential is given by $V(r)=k_{B} T(r / \sigma)^{-36}$, where $\sigma$ is the particles' center-to-center distance. $k_{B} T=1$ and the average radius of a host-liquid particle, $a=1$, set the units of energy and length. All particles have the same mass, $m=1$. The solvent friction coefficient, which also sets the Langevin forces, is fixed to $\zeta_{0}=50$ in these units. The near-equilibrium dynamics of this system has been analyzed in detail before, 15116 establishing a mode-coupling glass transition at an overall packing fraction $\varphi \approx 0.595$. At the highest packing fraction investigated here, $\varphi=0.62$ the system cannot be equilibrated in the available computer time; here we report measurements obtained after a waiting time $t_{w}=2.5 \times 10^{4}$ after setting up the simulation runs. For forces exceeding $F \approx 35 k_{B} T / a$, this is long enough to obtain results that do not depend on $t_{w}$; for smaller forces, aging effects may still affect the data at this highest packing fraction.

The simulation box is 8 times longer in the direction of the applied force than in the transversal directions, to allow the study of longer trajectories of the tracer, and contains 1000 particles, as shown in the snapshot in Fig. 11. At the highest density studied here, $\varphi=0.62$, the width of the box is $\approx 9.5 a$, enough to relax the structural effects caused by the moving probe. At time $t=0$, one particle is selected at random to be the probe particle (hence, the probe radius $a_{s}=a=1$ ), and a constant external force $\vec{F}^{\mathrm{ex}}=\left(F^{\mathrm{ex}}, 0,0\right)$ is exerted over it (in addition to the interparticle, friction and Brownian forces), and its trajectory is monitored. When the tracer has travelled a distance equal to half the box length, a new probe is selected from scratch, and the average velocity is obtained from the average trajectory. The simulation scheme is identical to the one used recently ${ }^{[14}$ For small forces, trajectories were recorded up to $t=10000$, before selecting a new probe. In these cases,
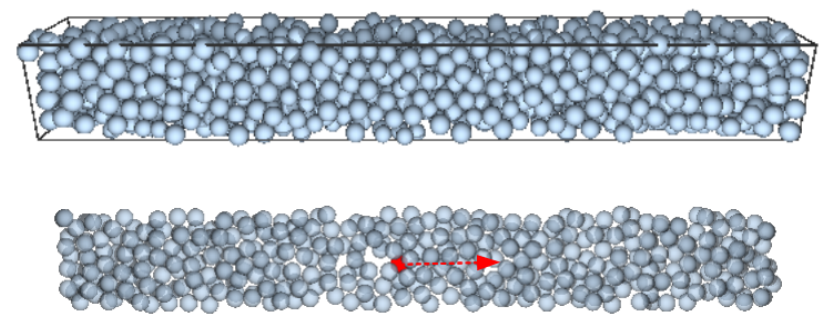

Fig. 1 Snapshot of the simulated system at packing fraction $\varphi=0.55$, showing the box geometry and an examplary run with a randomly selected probe. An arrow indicates the direction of the applied force.

sampling was performed over 300 trajectories obtained from different initial conditions and tracer particles.

From the simulation runs we thus obtain the probe response in terms of the friction coefficient $\zeta\left(F^{\mathrm{ex}}\right)$ at various packing fractions, using the steady-state relationship, Eq. (1). We also analyze the dynamics of density fluctuations, characterized by the tagged-particle density correlation functions $\phi_{\vec{q}}^{s}(t)=\left\langle\rho^{s *}(\vec{q}, t) \rho^{s}(\vec{q})\right\rangle$. Here, $\rho^{s}(\vec{q})=\exp \left[i \vec{q} \vec{r}_{s}\right]$ is the taggedparticle density fluctuation to wave vector $\vec{q}$. In an isotropic homogeneous liquid, the correlation function depends on the wave vector only through $q=|\vec{q}|$; however, in the present context, application of the external force degrades this spherical symmetry to a merely rotational one. As above, angular brackets denote ensemble averaging; here, however we distinguish equilibrium from steady-state averages, leading to, respectively, transient and stationary correlation functions.

\section{Schematic Models}

In this section, we summarize the main equations defining the schematic models. Mode-coupling theory (MCT) in the integration-through transients framework uses temporally and spatially resolved transient density correlation functions to describe the nonlinear response of the system to the strong external perturbation. In the case of constant-forcing active microrheology, the transient tagged-particle density correlation function $\phi_{\vec{q}}^{s}(t)$ is the basic dynamical quantity. Acknowledging the symmetry-breaking direction of the external force, it will be useful to distinguish for the probe particle the directions $\vec{q} \| \vec{F}^{\text {ex }}$ and $\vec{q} \perp \vec{F}^{\text {ex }}$. If $\vec{q}$ has a component parallel to the force, the displacement of the particle due to the external force causes the correlator to become complex valued, as it is the Fourier transform of a tagged-particle density profile that is not centered on the origin. Formally, this is a consequence of the non-Hermitian time-evolution operator in the Smoluchowski equation without detailed balance. If $\vec{q}$ is perpendicular to $\vec{F}^{\text {ex }}$, the correlator stays real. The internal forces acting on the tracer are determined by tracer- and host-liquid den- 
sity fluctuations. Whitin MCT, the latter are captured by the intermediate scattering function, $\phi_{q}(t)$, which, in an appropriate thermodynamic limit can be taken as the equilibrium one (being real and exhibiting full rotational symmetry).

The schematic model describes the dynamics of the system through wave-number independent functions $\phi(t)$ and $\phi_{\alpha}^{s}(t)$ that serve as proxies for the full correlation functions; here, $\alpha \in\{\|, \perp\}$ tells apart a complex- and a real-valued tracer correlator. The neglect of spatial resolution is motivated by equilibrium MCT, where a factorization theorem states that all length scales are strongly coupled and arrest simultaneously at the MCT transition. ${ }^{[17}$ The two kinds of tracer correlators capture the dominant effects of the external force: some correlators turn complex, while others remain (almost) real. The non-Markovian equations of motion analogous to the microscopic Mori-Zwanzig equations read

$$
\begin{gathered}
\partial_{t} \phi(t)+\Gamma\left\{\phi(t)+\int_{0}^{t} m\left(t-t^{\prime}\right) \partial_{t^{\prime}} \phi\left(t^{\prime}\right) d t^{\prime}\right\}=0, \\
\partial_{t} \phi_{\alpha}^{s}(t)+\omega_{\alpha}^{s}\left\{\phi_{\alpha}^{s}(t)+\int_{0}^{t} m_{\alpha}^{s}\left(t-t^{\prime}\right) \partial_{t^{\prime}} \phi_{\alpha}^{s}\left(t^{\prime}\right) d t^{\prime}\right\}=0 .
\end{gathered}
$$

Here, $\Gamma$ models the short-time dynamics of the host-liquid particles; we choose time units such that $\Gamma=1$. The short-time dynamics of $\phi_{\alpha}^{s}(t)$ is affected by the non-Hermitian nature of the underlying time-evolution operator and is modeled by $\omega_{\|}^{s}=\Gamma_{s}\left(1-i \kappa_{0} F^{\mathrm{ex}}\right)$ and $\omega_{\perp}^{s}=\Gamma_{s}$. The coefficient $\Gamma_{s}$ could be used to describe a short-time diffusion coefficient of the probe that differs from that of the host-liquid particles; we set $\Gamma_{s}=1$ for simplicity. The strength of the external force relative to internal ones at short times is measured by $\kappa_{0}$.

The memory kernels $m(t)$ and $m_{\alpha}^{s}(t)$ are approximated in MCT as nonlinear functionals of the density correlators themselves. We take for the schematic model

$$
\begin{gathered}
m(t)=v_{1} \phi(t)+v_{2} \phi(t)^{2} \\
m_{\|}^{s}(t)=\left[v_{1}^{s} \phi_{\|}^{s *}(t) \phi(t)+v_{2}^{s} \phi_{\perp}^{s}(t) \phi(t)\right] /\left(1-i \kappa_{\|} F^{\mathrm{ex}}\right), \\
m_{\perp}^{s}(t)=\left[v_{1}^{s} \phi_{\perp}^{s}(t) \phi(t)+v_{2}^{s} \Re \phi_{\|}^{s}(t) \phi(t)\right] /\left(1+\left(\kappa_{\perp} F^{\mathrm{ex}}\right)^{2}\right) .
\end{gathered}
$$

All coupling parameters $v_{i}$ and $v_{i}^{s}$ are taken to be real and positive; they model force-free equilibrium structural correlations. Equation (4) just specifies the well-known $\mathrm{F}_{12}$ model that is regularly used to analyze linear-response dynamics of glass forming liquids within mode-coupling theory. ${ }^{[17}$ The quadratic polynomial mimics the feedback mechanism termed "cage effect' that causes the slowing down in the structural relaxation of the host liquid. The bilinear coupling to $\phi_{\alpha}^{s}(t)$ and $\phi(t)$ in Eqs. (5) and (6) mimics that internal forces on the probe relax via its motion and via rearrangements of the surrounding particles. Both tracer motion parallel and perpendicular to the external force can relax the local friction. Spatial inversion symmetry demands that $\phi_{\|}^{s}(t)$ and $\phi_{\|}^{s *}(t)$ are coupled, which is assured by the complex conjugate appearing in $m_{\|}^{s}(t)$. The ensuing force driven delocalization transition can be mapped out using the external force as single relevant control parameter. ${ }^{14}$ Since $m_{\perp}^{s}(t)$ is real-valued, only the real part $\Re \phi_{\|}^{s}(t)$ can enter in Eq. [6. The external force enters differently in $m_{\|}^{s}(t)$ and $m_{\perp}^{s}(t)$ : the kernel $m_{\|}^{s}(t)$ is complexvalued, and $F^{\mathrm{ex}}$ suppresses both parts, while $m_{\perp}^{s}(t)$ needs to stay real-valued. Including $F^{\mathrm{ex}}$ in the denominator ensures the correct physical behavior that large forces decrease the coupling strength. Equations (5) and (6) explicitly express the symmetry under inversion of the force: $\phi_{\|}^{s}(-F)=\phi_{\|}^{s *}(F)$ but $\phi_{\perp}^{s}(-F)=\phi_{\perp}^{s}(F)$. The positive parameters $\kappa_{\|}$and $\kappa_{\perp}$ measure the effective force in the directions dominantly parallel and dominantly perpendicular to the external force. They should, by analogy to the microscopic model, be smooth functions of the thermodynamic control variables,, 18 and we take for simplicity $\kappa_{\|}=\kappa_{0}$ which was the choice in the original schematic model. 14

Finally, the friction coefficient is expressed through dynamical correlation functions via a nonequilibrium generalization of the Green-Kubo relation central to the ITT framework $\cdot{ }^{14}$ Following a mode-coupling approximation, we arrive at, schematically, $\zeta=\zeta_{0}+\Delta \zeta$,

$$
\Delta \zeta / \zeta_{0}=\mu_{\|} \Gamma_{s} \int_{0}^{\infty} d t \Re \phi_{\|}^{s}(t) \phi(t)+\mu_{\perp} \Gamma_{s} \int_{0}^{\infty} d t \phi_{\perp}^{s}(t) \phi(t),
$$

where the parameters $\mu_{\alpha} \geq 0$ replace angular-dependent coupling coefficients that are given by the equilibrium structure functions in the microscopic theory. Again this MCT approximation expresses that the friction on the probe arises from the cumulated transient fluctuations of probe and host-fluid densities. From the correlation function $\alpha=\|$, only the real part enters due to spatial inversion symmetry. Equations (2) to (7) specify our schematic model completely. They can further be motivated by an ad-hoc simplification of the full microscopic MCT, restricting wave-vector integrals to four wave vectors, $\vec{q} \| \vec{F}^{\text {ex }}$ and $\vec{q} \perp \vec{F}^{\text {ex }} \cdot \frac{18}{18}$ For each $\vec{q}$ also $-\vec{q}$ has to be taken to obey the required symmetry $\phi_{-\vec{q}}^{s}(t)=\phi_{\vec{q}}^{s *}(t)$; this justifies the appearance of the complex conjugate in (5).

The earlier schematic model for active microrheology 14 only included a single complex correlator, concentrating on the novel transition that a particle is pulled mobile by a finite external force. The present three-correlator model reduces to this two-correlator model upon setting $v_{2}^{s}=\mu_{\perp}=0$. Also, for $F^{\mathrm{ex}}=0$, we obtain $\phi_{\|}^{s}(t)=\phi_{\perp}^{s}(t)$ and the model reduces to the Sjögren model of tagged-particle dynamics in the nearequilibrium glass forming liquid, with a single probe-coupling parameter $v^{s}=v_{1}^{s}+v_{2}^{s}$; the latter is a standard model for analysis of linear-response dynamics of glass forming liquids, e.g., in terms of scattering spectra. 17 
The present model additionally captures a nontrivial limit of the friction at high force, $\Delta \zeta_{\infty} \equiv \Delta \zeta\left(F^{\mathrm{ex}} \rightarrow \infty\right)$. The model with $\mu_{\perp}=0$ predicts $\Delta \zeta_{\infty}=0$ as the presence of the term $\propto$ $i F^{\mathrm{ex}}$ in $\omega_{\|}^{s}$ leads to arbitrarily fast oscillations in $\phi_{\|}^{s}(t)$; in the integral determining $\Delta \zeta$ these lead to cancellations such that the overall integral vanishes. Only when $\mu_{\perp} \neq 0$ does one get an additional contribution to $\Delta \zeta$ in the large force limit. If we furthermore neglect all memory kernels, we obtain as the low-density (weak-coupling) limit of the schematic model the ratio $\Delta \zeta_{\infty} / \Delta \zeta\left(F^{\mathrm{ex}} \rightarrow 0\right)=\mu_{\perp} /\left(\mu_{\|}+\mu_{\perp}\right)$. This ratio is known to equal $1 / 2$ exactly in the low-density limit 19 , checked also in our simulations. We thus, for simplicity, set $\mu_{\|}=\mu_{\perp}=\mu$ throughout in the following.

\section{Data Analysis}

The schematic model presented above has several fit parameters. Two of them, $v_{1}$ and $v_{2}$, do not depend on the probe at all; they specify the dynamics of the host liquid and its vicinity to the glass transition which occurs for some critical coupling $\left(v_{1}^{c}, v_{2}^{c}\right)$. Thus, these two parameters can in principle be determined independently. In the cases we analyze, the only physical control parameter is the packing fraction of the suspension, $\varphi$. One demands that the fit parameters follow a linear relationship $\left(v_{1}, v_{2}\right)=\left(v_{1}^{c}, v_{2}^{c}\right)(1+\varepsilon)$ with $\varepsilon \propto\left(\varphi-\varphi^{c}\right) / \varphi^{c}$, where $\varphi^{c}$ is the glass-transition packing fraction of the suspension. The critical point is then fixed by demanding the MCT exponents of the schematic model to match those found in experiment or simulation. In practice, this fixes the pair $\left(v_{1}^{c}, v_{2}^{c}\right)$, and one fits $\varepsilon$ for each packing fraction, biasing it to follow the stated linear relationship.

Two parameters $v_{1}^{s}$ and $v_{2}^{s}$ describe the coupling strength of the probe to the host suspension; their sum $v^{s}$ could be determined from analyzing linear-response measurements independently. In principle, they depend on the density of the host; we will in the following thus allow $v^{s}$ to increase with increasing packing fraction. Inspection of the microscopic model and its symmetries $\frac{18}{18}$ suggests the fixed ratio $v_{1}^{s} / v_{2}^{s}=2$. The remaining parameters $\kappa_{\perp}$ and $\kappa_{\|}$are global parameters (fixed differently for the simulation and for the experiment to reflect the difference in systems) specifying the relative influence of the external force on the memory kernel.

In our fits of both BD simulation and experimental data, we aimed at keeping as many parameters fixed as is physically plausible. First, we set $v_{2}^{c}=2$, resulting in $v_{1}^{c}=2(\sqrt{2}-1)$, which renders the near-equilibrium asymptotic behavior of the schematic model close to that observed for the hard-sphere glass transition. Furthermore, $\varepsilon(\varphi)$ is estimated from the known linear-response regime for the simulation data; for the fits to the experimental data we require that the obtained values of $\varepsilon$ are of the same magnitude for $\varphi$ not too close to the

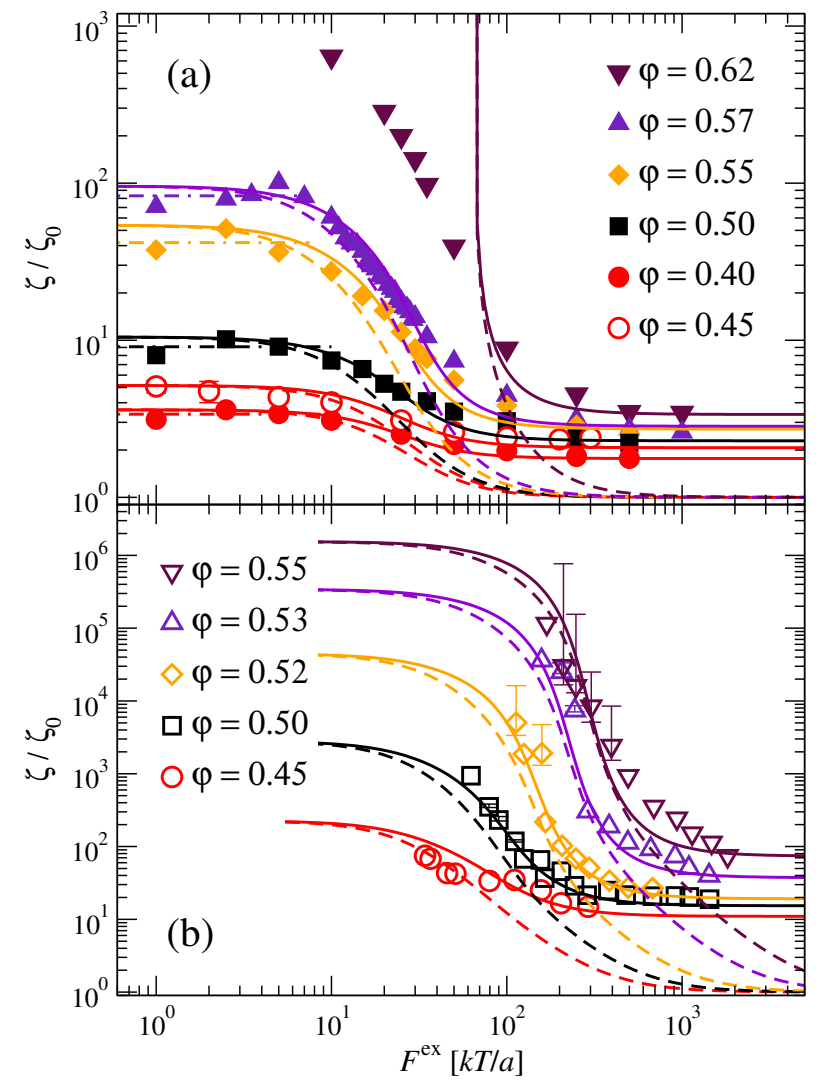

Fig. 2 Microscopic friction coefficients $\zeta$ as a function of external force $F^{\mathrm{ex}}$ : symbols are (a) Brownian dynamics (filled) and hard-sphere simulations of Ref. 20](open); (b) experiment of Ref. 12. evaluted from the original velocity data (Fig. 3) and translated to dimensionless quantities by setting $\zeta_{0}=0.095 \mathrm{mg} / \mathrm{s}$ and $1 \mathrm{pN}=271.9 k_{B} T / a$. Lines are fits with schematic models of mode-coupling theory, taking only fluctuations in direction of the force into account (dashed), or also in the perpendicular direction (solid lines). Horizontal dot-dashed bars in (a) give the quiescent self-diffusion coefficients.

critical point. To capture the density dependence of the highforce plateau, we also increase $\mu$ with increasing density.

We first turn to a discussion of the friction coefficient $\zeta$, shown in Fig. 2 for our Brownian dynamics simulations, and for the experiment by Habdas et al. 12 . We have translated the latter from the reported velocity data and into dimensionless units by using the known solvent properties, leading to $\zeta_{0}=0.095 \mathrm{pNs} / \mu \mathrm{m}$. In Fig. 2, also a result from simulations of monodisperse hard spheres by Carpen and Brady 20 is shown, highlighting that our choice of soft-sphere potential and polydispersity are not crucial for the present discussion. While in both simulations, probe and host-liquid particles were of the same size, $\delta=a_{s} / a=1$, the quoted values for the experiment in Ref. 12 are $a_{s}=2.25 \mu \mathrm{m}$ and $a=1.1 \mu \mathrm{m}$, 
leading to a size ratio of $\delta \approx 2.05$. This difference explains a shift in force scales when comparing the two sets of data.

Solid lines in Fig. 2 are fits using the schematic model. Fit parameters are given in Table 11, additionally, under the constraint that the fits describe reasonably the known (simulation) or anticipated (experiment) linear response, we found $\left(\kappa_{\|}, \kappa_{\perp}\right)=(0.25,0.0625) a / k_{B} T$ for the simulation, and $\left(\kappa_{\|}, \kappa_{\perp}\right)=(0.046,0.0115) a / k_{B} T$ for the experimental data to give satisfying results. These are not the only parameter sets that give reasonable fits; on the schematic level, no more physical significance can be attached to the choice of parameters.

As is evident, the model reproduces the qualitative features of the available data, in particular the steep change of $\zeta$ already in the liquid around a threshold of $O\left(20 k_{B} T / a\right)$ for the simulation and $O\left(200 k_{B} T / a\right)$ for the experiment (larger due to the larger force required to pull free a larger probe). As pointed out earlier, 14 this threshold force is in the schematic model precisely defined as the force that is needed to pull the probe particle free even if the suspending host is glassy. Reassuringly, microscopic calculations for the hard-sphere model yielded values comparable to the ones found in our simulation. From the schematic model, we get for the critical force just at the glass transition $F_{c}^{\mathrm{ex}} \approx 63 k_{B} T / a$ for the simulation, and $F_{c}^{\mathrm{ex}} \approx 800 k_{B} T / a \approx 3 \mathrm{pN}$ for the experiment. At higher densities, even larger forces are needed to break the cages. According to MCT, the host system is in an ideal-glass state for densities $\varphi>\varphi^{c} \approx 0.595$ in the simulation; hence, at vanishing external force, the single-particle friction coefficient is infinite since the tracer remains localized in the glass. The simulation data for $\varphi=0.62$ still show finite values of $\zeta$ at all forces, which may partly be due to aging effects; it may also reflect deviations of the simulated glass from the idealized MCT description.

Before turning to a discussion of the large-force behavior, let us briefly discuss the steady-state probe velocities corresponding to Fig. 2. This is the quantity originally obtained in the experiment, and is shown in Fig. 3. The plot highlights the difficulty to stay within the linear-response regime in dense colloids. Velocities smaller than $10^{-3} k_{B} T / a \zeta_{0} \sim 10^{-3} D_{0} / a$ have to be measured, where $D_{0} \sim \mu \mathrm{m}^{2} / \mathrm{s}$ is a typical short-time diffusion coefficient for a colloidal particle of radius $a \sim 1 \mu \mathrm{m}$. The resulting velocities are on the order of a few particle diameters per week, which clearly is a challenge. Such small velocities pose the additional problem of ensuring a typical enough sample of the configuration space required in the ensemble average, a problem also for the simulations.

In both Figs. 2 and 3 , dashed lines represent the predictions of the schematic model when setting $\mu_{\perp}=0$, i.e., ignoring the contribution of fluctuations perpendicular to the external force to the friction coefficient. Fits with somewhat different parameters also setting $v_{2}^{s}=0$ have been presented earlier; 14 they are qualitatively the same. The resulting curves still show the

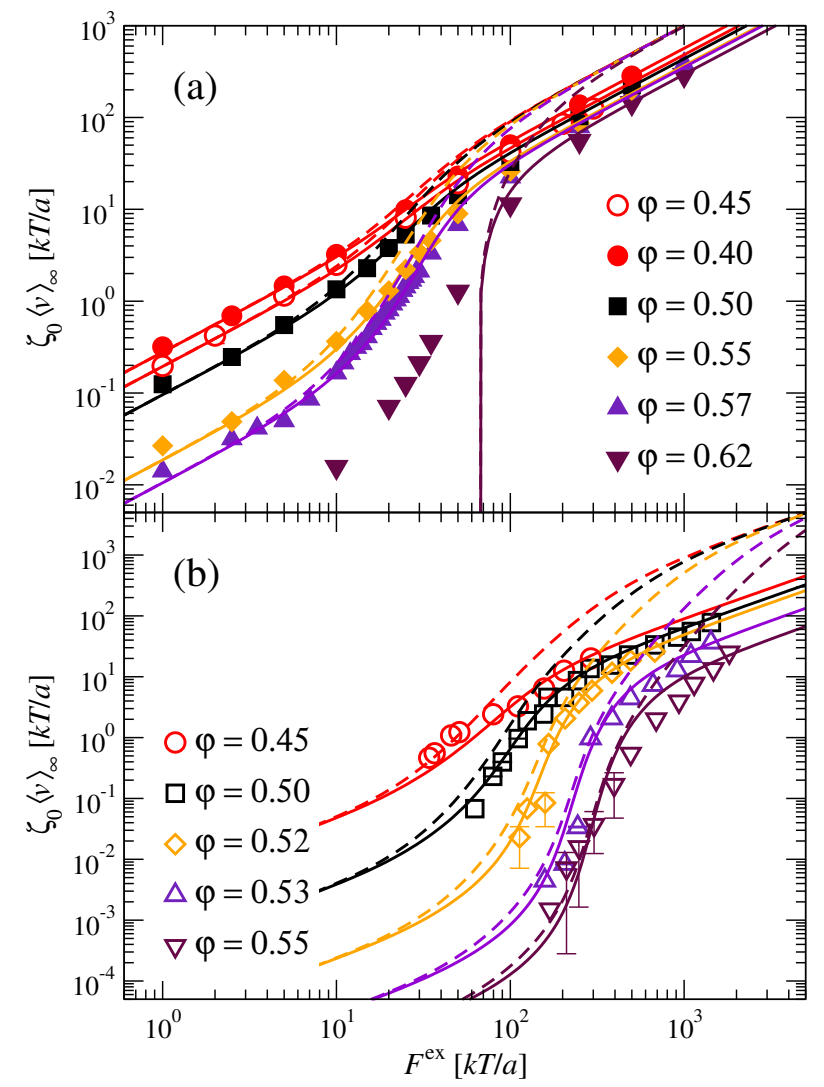

Fig. 3 Steady-state probe velocities $\langle\vec{v}\rangle_{\infty}$ corresponding to Fig. 2 as a function of external force: computer-simulation (a) and experiment (b). Lines are the same schematic-model fits as in Fig. 2

generic features of the rapid decrease in friction in the vicinity of the force threshold. The most striking difference is that for $\mu_{\perp}=0$, one obtains $\zeta\left(F^{\mathrm{ex}} \rightarrow \infty\right)=\zeta_{0}$, the free-particle friction coefficient, as explained above. This behavior is not supported by the data, which clearly show a larger, densitydependent high-force plateau in $\zeta\left(F^{\mathrm{ex}}\right)$.

At least within the schematic model, including fluctuations perpendicular to the direction of the external force restores the nontrivial high-force plateau. Here, $\phi_{\perp}^{s}(t)$ remains a realvalued positive function whose decay is fixed by the equilibrium short-time relaxation of the probe particle. Consequently, a finite contribution remains for $\Delta \zeta$ even as $F^{\text {ex }} \rightarrow \infty$.

A comparison between simulation and experiment regarding the high-force plateau in $\zeta\left(F^{\mathrm{ex}}\right)$ is instructive. Replotting the experimental data as in Fig. 2 reveals that the data is not yet in the limiting regime $F^{\mathrm{ex}} \rightarrow \infty$ for all densities, but our schematic-model fits suggest a confident extrapolation. We obtain the values shown in Fig. 4 as symbols. The difference between the two data sets is the result of two physical differences in the systems: the ratio $\delta=a_{s} / a$ and the influence of hydrodynamic interactions. To demonstrate this, we have in- 


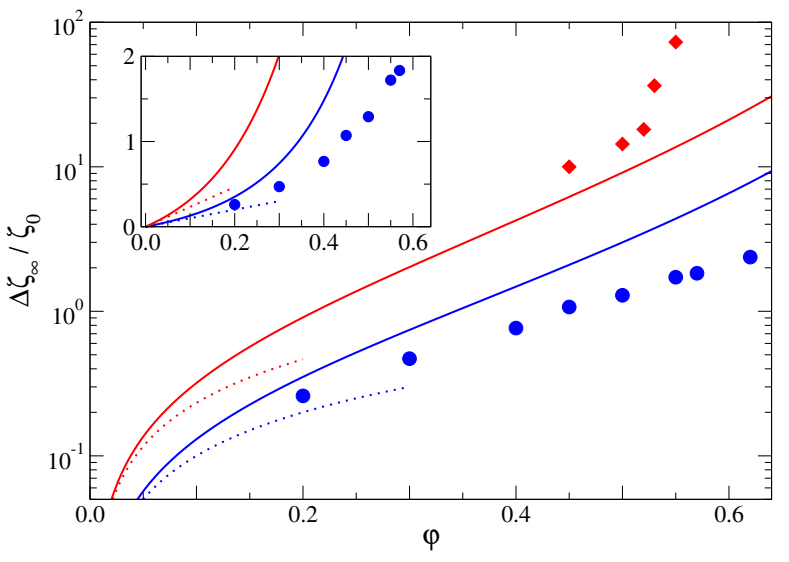

Fig. 4 High-force limit of the microscopic friction increment, $\Delta \zeta_{\infty} / \zeta_{0}=\zeta\left(F^{\mathrm{ex}} \rightarrow \infty\right) / \zeta_{0}-1$, obtained from simulation (circles; size ratio $\delta=a_{s} / a=1$ ) and experiment (squares; $\delta=2.05$ ), as a function of packing fraction $\varphi$. Lines are the low-density estimates (dotted) by Squires and Brady, ${ }^{19}$ corrected for packing effects by the equilibrium contact value from the BMCSL equation of state for these size ratios (solid lines).

cluded in Fig. 4 as dotted lines the result obtained by Squires and Brady. 19 Evaluating the hydrodynamic equations for $\varphi \rightarrow$ 0 , they obtained $\Delta \zeta_{\infty} / \zeta_{0}=(1 / 4) \varphi(1+\delta)^{2}$. A first-order account for packing effects in the host liquid is obtained by replacing the packing fraction $\varphi$ in this expression by $\varphi g_{d}(\varphi)$, where $g_{d}(\varphi)$ is the probe-host-particle contact value. For the latter, we use the form corresponding to the BMCSL equation of state for hard-sphere mixtures due to Grundke and Henderson, 21 in the limit of vanishing probe-particle density, $g_{d}(\varphi)=$ $(1-\varphi+3 \varphi \delta /(1+\delta)) /(1-\varphi)^{2}+2 \varphi^{2}(\delta /(1+\delta))^{2} /(1-\varphi)^{3}$. Note that this expression differs slightly from the more common Carnahan-Starling result for $\delta=1$, used, e.g., by Carpen and Brady. ${ }^{20}$ As shown in Fig. 4, this theory describes reasonably well the experimental data when we set $\delta=2.05$. The increase due to size-ratio effects alone is about a factor 4 at

Table 1 Fit parameters for the MCT schematic model

\begin{tabular}{llll}
\hline & $\varepsilon$ & $v_{s}$ & $\mu$ \\
\hline $\operatorname{BD} \varphi=0.40$ & -0.98 & 9 & 0.45 \\
$\operatorname{HS} \varphi=0.45$ & -0.87 & 9 & 0.59 \\
$\operatorname{BD} \varphi=0.50$ & -0.54 & 9.75 & 0.62 \\
$\operatorname{BD} \varphi=0.55$ & -0.23 & 11.25 & 0.74 \\
$\operatorname{BD} \varphi=0.57$ & -0.185 & 14.25 & 0.78 \\
$\operatorname{BD} \varphi=0.62$ & 0.185 & 33 & 0.93 \\
$\exp \varphi=0.45$ & -0.17 & 4.5 & 14.3 \\
$\exp \varphi=0.50$ & -0.07 & 7.5 & 20 \\
$\exp \varphi=0.52$ & -0.03 & 15 & 25 \\
$\exp \varphi=0.53$ & -0.02 & 30 & 50 \\
$\exp \varphi=0.55$ & -0.015 & 45 & 100 \\
\hline
\end{tabular}

6 | Journal Name, 2010, [vol],1-8

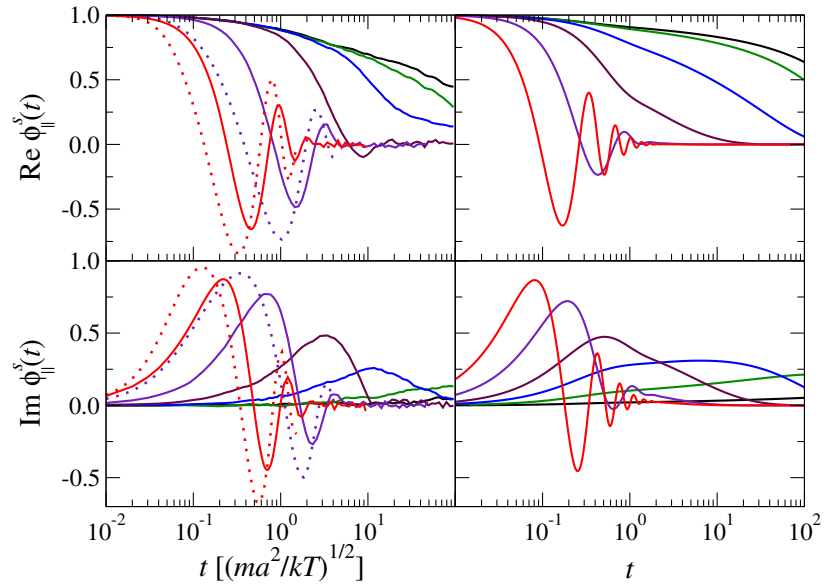

Fig. 5 Probe-density correlation functions $\phi_{\|}^{s}(t)$ from BD simulations at $q=3.85 / a$ and $\varphi=0.55$ (left) and from the corresponding schematic model fit (right). Top (bottom) panels show the real (imaginary) part of the correlators. For the simulation, $a F^{\mathrm{ex}} /\left(k_{B} T\right)=1,5,15,35,100$, and 250 (right to left).

$\varphi \approx 0.5$. The contact-value-corrected low-density expression for $\delta=1$ exceeds the BD results; the difference might be due to the polydispersity and the slightly soft potential used in the simulations, as both factors reduce $g_{d}(\varphi)$. At $\varphi<0.2$, the simulation data approaches the low-density asymptote.

It is worth pointing out that the friction coefficient at small forces, $F^{\mathrm{ex}} \rightarrow 0$, shows completely different scaling than the one for $F^{\mathrm{ex}} \rightarrow \infty$ : while the latter is well described by hydrodynamics corrected for pair-density effects, the former is governed by the approach of the equilibrium system to the glass transition, where $\zeta\left(F^{\mathrm{ex}} \rightarrow 0\right) \rightarrow \infty$ as $\varphi \rightarrow \varphi^{c}$ from the liquid side. This is clearly demonstrated by the data shown in Fig. 2. We have checked that at low densities, $\Delta \zeta\left(F^{\mathrm{ex}} \rightarrow 0\right) / \Delta \zeta\left(F^{\mathrm{ex}} \rightarrow \infty\right)=2$ as predicted ${ }^{19 \mid 20}$ also holds for our simulations (up to about $\varphi \approx 0.2$ ). Clearly, at higher density $\Delta \zeta\left(F^{\mathrm{ex}} \rightarrow 0\right)$ rises much more dramatically than the $F^{\mathrm{ex}} \rightarrow \infty$ friction coefficient.

The strength of the MCT model is that it can not only explain the available data for the friction coefficient or steadystate velocity, but also the underlying probe dynamics in terms of the tagged-particle density correlation functions. We have obtained both the stationary and, for some values of $F^{\mathrm{ex}}$, also the transient correlation functions from the BD simulation; both are shown in the left-hand panels of Figs. 5 and 6 as continuous and dotted lines, respectively, for a wave number $q a=3.85$, packing fraction $\varphi=0.55$ and various external forces. In Fig. 5, the alignment $\vec{q} \| \vec{F}^{\text {ex }}$ has been chosen, while Fig. 6 shows $\vec{q} \perp \vec{F}^{\text {ex }}$. Even at the largest forces we investigate, the difference between stationary and transient correlation functions is not qualitative. In the direction of the force, the transient correlation function decays faster than the sta- 


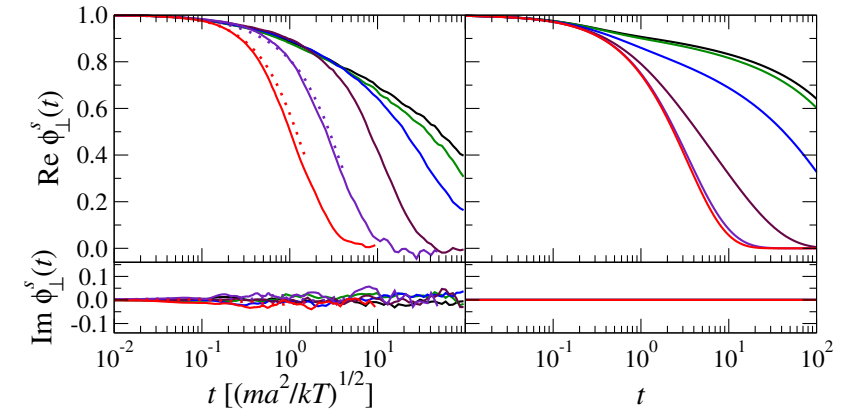

Fig. 6 Probe-density correlation functions $\phi_{\perp}^{s}(t)$ from BD simulations at $q=3.85 / a$ and $\varphi=0.55$ (left) and from the corresponding schematic model fit (right). Top (bottom) panels show real (imaginary) parts of the correlators for forces as in Fig. 5

tionary one. We interpret this as being due to a "wave front", i.e., a region of increased density building up in front of the pulled particle, which slows down the structural rearrangement in this direction once it is established in the steady state (see also the discussion in Ref. 19). Just after applying the external force, this wave front has not been built up for short times, and this is probed by the transient correlation function.

We now focus on the stationary correlation functions that are easier to obtain from the simulation, since the difference to the transient quantity is not important for our purposes. In mode-coupling theory based on integration through transients, the transient correlation functions are the central quantities, and the evolution towards the stationary functions needs to be understood separately. ${ }^{[22]}$ Thus, the schematic-model correlators that are shown in the right-hand panels should, strictly speaking, be interpreted as transient correlation functions.

One recognizes a number of generic features in the correlation functions, that are present in the simulation and correctly reproduced by our schematic model. First, $\phi_{\|}^{s}(t)$ is a complex quantity, and it shows pronounced oscillations at large $F^{\text {ex }}$ that are indicative of a probe moving with a finite velocity that is faster than the diffusive exploration of its configuration space. 14 For small external forces, the correlation functions stay close to the equilibrium tagged-particle correlator recovered for $F^{\mathrm{ex}} \rightarrow 0$, indicating the linear-response regime. As $F^{\mathrm{ex}}$ is increased, the slow relaxation of the correlator is increasingly accelerated, which is the microscopic origin for the pronounced force thinning observed in Figs. 2 and 3 .

Taking $\vec{q} \perp \vec{F}^{\text {ex }}$, one instead recovers a real-valued correlation function. Figure 6 confirms this prediction for the BD computer simulation: the calculated imaginary part is zero within the noise level. Here, too, large forces lead to a shortening of the equilibrium slow relaxation time, but no oscillations are seen, as the probe particle does not perform net motion in the direction perpendicular to the force. At very high $F^{\mathrm{ex}}$, the schematic model predicts a saturation, where

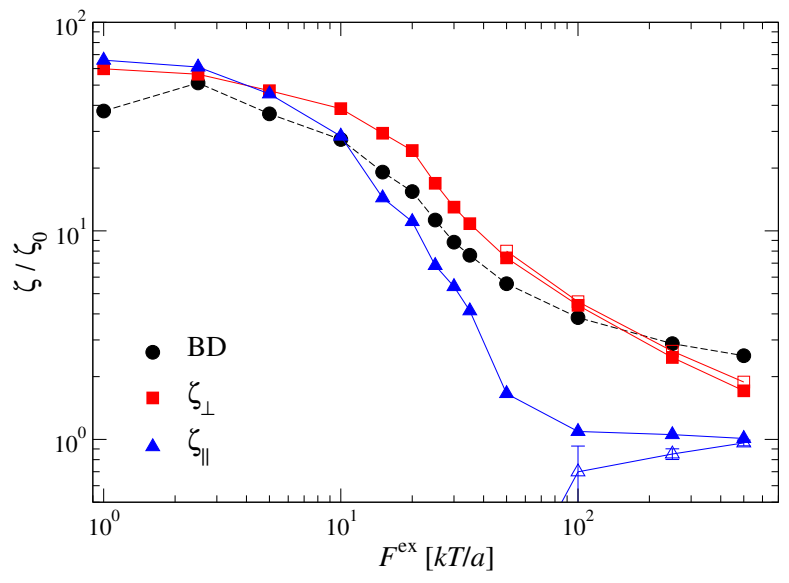

Fig. 7 Microscopic friction $1+\Delta \zeta\left(F^{\mathrm{ex}}\right) / \zeta_{0}$ for the BD simulation at $\varphi=0.55$. Circles: simulation data from Fig. 2, squares (triangles): contributions in Eq. (1) coming only from the simulated $\vec{q} \perp \vec{F}^{\text {ex }}$ $\left(\vec{q} \| \vec{F}^{\mathrm{ex}}\right)$ correlation functions shown in Figs. 5 and 6 Filled (open) symbols use the stationary (transient) correlation functions.

$\phi_{\perp}^{s}(t)$ approaches an exponential decay on the short-time diffusion scale. In the simulation, this is not (yet) clear. The integral over this remaining force-independent decay is, in the schematic model, the cause for the non-vanishing high-force friction increment over the solvent friction. The model thus predicts the large-force friction to be dominated by details of the short-time motion, but not by the slow structural relaxation. The good quality of the low-density predictions for $\Delta \zeta_{\infty}$ shown in Fig. 4 supports this.

Further support for the schematic-model interpretation assigning distinct roles to the principal directions $\vec{q} \perp \vec{F}^{\text {ex }}$ and $\vec{q} \| \vec{F}^{\text {ex }}$ at large external forces comes from the simulation data in Figs. 5 and 6 . In the microscopic theory, Eq. (7) is replaced by a wave-vector dependent integral over $\phi_{\vec{q}}^{s *}(t) \phi_{q}(t)$, taking into account fluctuations in all directions relative to the force. In principle, this integral could be evaluated with simulation data for the (transient) correlation functions, without making use of the MCT equations of motion. It is a formidable task to sample enough correlation functions with sufficient statistics in the simulation. To give a qualitative impression, we only evaluate the integral over the simulated correlators for the two particular wave vectors shown above separately; this corresponds to the two contributions in the schematic Eq. (7). The host-liquid correlation function needed in this equation has also been evaluated at the same wave number, $q=3.85 / a$, in the BD simulation. The result is shown in Fig. 7 for an exemplary packing fraction. The integral using $\vec{q} \| \vec{F}^{\mathrm{ex}}$ (triangle symbols) gives $\zeta \approx \zeta_{0}$ at large forces, i.e. $\Delta \zeta\left(F^{\mathrm{ex}}\right) \rightarrow 0$. Also in the BD simulation, the oscillations in $\phi_{\vec{q}}^{s}(t)$ in the direction parallel to the external force cancel each other, and the main contribution to the friction at high external forces stems from 
density fluctuations to wave vectors perpendicular to the force (squares in the figure). Our data for the transient correlation functions is not of sufficient quality to be used in the integration, although this should strictly speaking be done. For the highest forces we simulated, we give a numerical estimate for the integral value according to Eq. (7) using the transient correlators (open symbols in the figure). The difference is not large (slightly negative values in some cases indicate the magnitude of the error in the integration procedure, due to cutting off at too early times). This further corroborates the point, that wave-vector contributions perpendicular to the external force are prominent in the large-force limit.

\section{Conclusions}

We have presented a schematic mode-coupling-theory analysis of active nonlinear microrheology investigating the dynamics of a probe particle pulled by a strong constant force through a glass-forming dense colloidal suspension. Extending previous analysis, 14 our schematic model captures the qualitatively distinct behavior of probe-density correlation functions for fluctuations in the direction of and perpendicular to the external force. The distinct features - complex-valued and at high force oscillating correlation functions in the direction of the force, in contrast to force-thinning real-valued functions perpendicular to $\vec{F}^{\text {ex }}$ - reflect the property that the probe-particle density distribution is biased in the direction of the force and attains a finite steady-state velocity superseeding the quiescent motion at high enough forces, while perpendicular to the force, no net motion occurs. These features are confirmed in Brownian dynamics simulations.

Within the schematic model, contributions to the microscopic friction coefficient $\zeta\left(F^{\mathrm{ex}}\right)$ coming from the different directions start being distinguishable upon entering the nonlinear regime. Both contributions drop sharply around a threshold that is large compared to forces induced by thermal fluctuations alone. They lead to pronounced force thinning and the eventual delocalization of the driven probe even from a glassy surrounding matrix, at a critical force $F_{c}^{\mathrm{ex}}$. The contribution to the friction from fluctuations parallel to the external force vanishes as $F^{\mathrm{ex}} \rightarrow \infty$, but the contribution from the perpendicular directions remains finite, giving rise to a non-trivial high-force plateau above the Stokesian pure-solvent value. The existence of such a plateau is in qualitative agreement with both simulations and experiment, and also with predictions from lowdensity expansions.

It remains difficult to compare the mechanism causing a nontrivial high-force plateau, $\Delta \zeta_{\infty}>0$, in our present MCT approach with the one by Squires and Brady, $\frac{19}{19}$ but the two descriptions share a number of apparent similarities. In Ref. 19. it arises from a singular boundary layer, where advection and diffusion (otherwise negligible far from the probe at high force) compete close to the particle; transport of fluid particles out of the boundary layer requires motion parallel to the probe surface, i.e., perpendicular to the force. In MCT, probe fluctuations transverse to the direction of the applied force need to be included. In either case, fast diffusive motion of the host fluid particles, characterized by the short-time self-diffusivity 19 or $\Gamma_{s}$ in the schematic model, dominates the high-force friction. Structural correlations, which would be characterized by the much smaller long-time diffusivity respectively the slow relaxation time of the correlators in Figs. 5 and 6, dominate the quiescent friction.

We thank J. F. Brady for valuable discussions. This work was supported by Deutsche Forschungsgemeinschaft, SFB TR6 project A7, Helmholtz-Gemeinschaft (HGF HochschulNachwuchsgruppe VH-NG 406), and Junta de Andalucía (P09-FQM-4938). We acknowledge partial funding by the Zukunftskolleg der Universität Konstanz and by the German Excellence Initiative.

\section{References}

1 P. Cicuta and A. Donald, Soft Matter, 2007, 3, 1449.

2 T. M. Squires, Langmuir, 2008, 24, 1147-1159.

3 Y. Kimura, J. Phys. Soc. Jpn., 2009, 78, 041005.

4 T. M. Squires and T. G. Mason, Annu. Rev. Fluid Mech., 2010, 42, 413438.

5 A. J. Levine and T. C. Lubensky, Phys. Rev. Lett., 2000, 85, 1774-1777.

6 A. W. C. Lau, B. D. Hoffman, A. Davies, J. C. Crocker and T. C. Lubensky, Phys. Rev. Lett., 2003, 91, 198101.

7 C. Wilhelm, Phys. Rev. Lett., 2008, 101, 028101.

8 L. Wilson, A. Harrison, A. Schofield, J. Arlt and W. Poon, J. Phys. Chem. $B, 2009, \mathbf{1 1 3}, 3806$.

9 I. Sriram, E. M. Furst, R. J. DePuit and T. M. Squires, J. Rheol., 2009, 53, 357-381.

10 I. Sriram, A. Meyer and E. M. Furst, Phys. Fluids, 2010, 22, 062003.

11 M. B. Hastings, C. J. Olson Reichhardt and C. Reichhardt, Phys. Rev. Lett., 2003, 90, 098302.

12 P. Habdas, D. Schaar, A. C. Levitt and E. R. Weeks, Europhys. Lett., 2004, 67, 477-483.

13 S. R. Williams and D. J. Evans, Phys. Rev. Lett., 2006, 96, 015701.

14 I. Gazuz, A. M. Puertas, Th. Voigtmann and M. Fuchs, Phys. Rev. Lett., 2009, 102, 248302.

15 Th. Voigtmann, A. M. Puertas and M. Fuchs, Phys. Rev. E, 2004, 70, 061506.

16 F. Weysser, A. M. Puertas, M. Fuchs and Th. Voigtmann, Phys. Rev. E, 2010, 82, 011504.

17 W. Götze, Complex Dynamics of Glass-Forming Liquids, Oxford University Press, 2009.

18 M. V. Gnann, Analysis of schematic models of mode-coupling theory for colloids in external fields, Diploma thesis, Universität Konstanz, 2009.

19 T. M. Squires and J. F. Brady, Phys. Fluids, 2005, 17, 073101

20 I. C. Carpen and J. F. Brady, J. Rheol., 2005, 49, 1483-1502.

21 E. W. Grundke and D. Henderson, Molec. Phys., 1972, 24, 269-281.

22 M. Krüger, F. Weysser and Th. Voigtmann, Phys. Rev. E, 2010, 81, 061506. 